\title{
Interference techniques in digital holography
}

\author{
Myung K Kim, Lingfeng Yu and Christopher J Mann \\ Department of Physics, University of South Florida, Tampa, FL 33620, USA \\ E-mail: mkkim@cas.usf.edu
}

Received 17 November 2005, accepted for publication 23 February 2006

Published 8 June 2006

Online at stacks.iop.org/JOptA/8/S518

\begin{abstract}
Interference techniques in digital holography are discussed and experimental results from each technique are presented. Numerical reconstruction algorithms for digital holography are reviewed. The angular spectrum method is seen to be particularly advantageous, with the ability to remove noise and unwanted holographic terms. The dual wavelength optical unwrapping technique offers an unambiguous method of removing $2 \pi$ phase discontinuity. Application of wavelength-scanning digital interference holography is used to obtain tomographic images with synthesized short coherence length.
\end{abstract}

Keywords: digital holography, interference imaging, phase contrast microscopy, optical tomography

(Some figures in this article are in colour only in the electronic version)

\section{Introduction}

Digital holography has been experiencing rapid development in recent years because of many technical advantages, including a number of interference techniques that are unavailable or difficult and cumbersome in conventional holography. The principle of holography was first suggested by Gabor in 1948, as a method of recording complete threedimensional information of an object wave [1]. With the later invention of the laser and the introduction of the off-axis technique in 1962 [2], holography quickly gained scientific recognition. The advances in digital imaging and computation technologies have now made it feasible and advantageous to replace the photochemical processing of conventional holography with CCD arrays and numerical computation. A digital hologram is created by the interference between a coherent object and reference beam, which is digitally recorded by a CCD camera and processed by computational methods to obtain the holographic images [3-5]. The digital hologram contains not only amplitude information of the object, but also phase [6,7]. Moreover, the ability of the CCD camera to quantify the recorded light gives rise to a number of post-processing methods that can for instance be used to calculate optical thickness or refractive index variations of an object provided knowledge of one or the other is available. Digital holography has been applied in diverse fields including metrology [8], deformation measurement [9], vibrational analysis [10] and more recently biological microscopy [11-14]. The applications to microscopy are particularly advantageous. Conventional bright-field microscopes have difficulty in observing transparent samples which exhibit little intensity contrast. The phase contrast microscopy technique of Zernike and differential interference contrast (DIC) microscopy of Nomarksi do not offer direct quantitative evaluation of the phase information. The unavailability of this information in these techniques presents a difficulty in observing and interpreting morphological changes and properties of a sample.

Digital holography not only offers quantitative phase information but high fidelity and high resolution images with precision of optical thickness on the order of tens of nanometres [15]. Another appealing aspect of the technique is numerical focusing, emulating the focusing control of conventional microscopes. In particular, the use of the angular spectrum reconstruction algorithm provides a significant advantage in focusing and reconstruction [16]. It has no minimum distance requirement from the object to the hologram plane and allows for flexible and effective filtering and control of the zero order and spurious noise components from sources such as stray reflections. A common presumption is that coherent imaging suffers from the image degrading effect of coherent noise; however, through careful control of the laser 

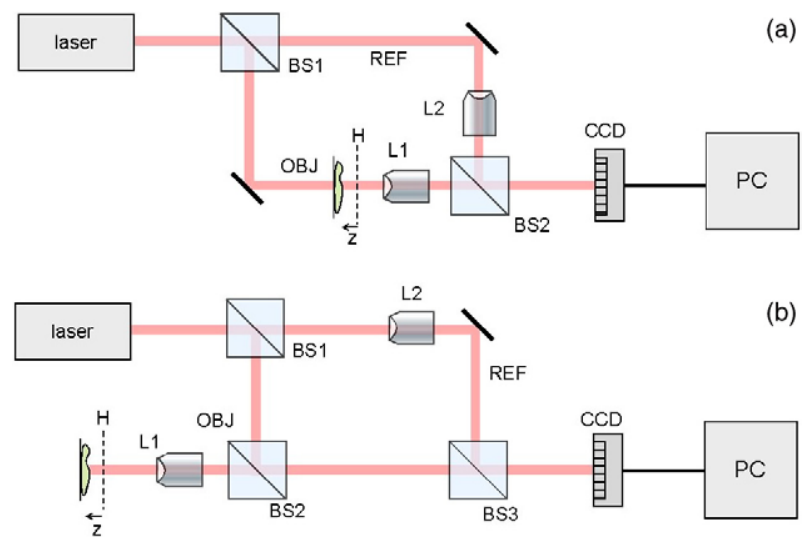

Figure 1. Digital holography experiments using transmission (a) and reflective (b) geometries.

beam and other optical quality, remarkably clean images can be obtained. This is especially true with phase imaging digital holography because of its relative immunity to amplitude or phase noise of the laser profile.

\section{Numerical diffraction: theory}

The digital holography experiments are performed in both transmission and reflective geometries as depicted in figure 1. The output from the laser, spatially filtered and collimated, is split into an object and reference beam in an interferometer based on the Mach-Zehnder configuration. The object specimen, mounted on an $x y z$-translation stage, is placed at a distance $z$ from the hologram plane $H$, which is imaged and magnified by the lens L1 and projected onto the CCD array. The reference beam is similarly magnified by lens L2 and the interference between the object and reference beams is recorded and stored onto a computer. Once the hologram has been recorded, it remains to reconstruct the optical field by computationally simulating diffraction using one of a number of numerical methods.

\subsection{Huygens convolution method}

The convolution approach represents a computationally heaviest form of holographic reconstruction with modest degree of accuracy [17]. The reconstructed complex wavefield $E(x, y)$ is found by

$$
\begin{aligned}
& E(x, y ; z)=-\frac{\mathrm{i} k}{2 \pi} \iint_{\Sigma} E_{0}\left(x_{0}, y_{0}\right) \\
& \quad \times \exp \left[\mathrm{i} k \sqrt{\left(x-x_{0}\right)^{2}+\left(y-y_{0}\right)^{2}+z^{2}}\right] \mathrm{d} x_{0} \mathrm{~d} y_{0} .
\end{aligned}
$$

This is a convolution:

$$
\begin{aligned}
E(x, y ; z) & =E_{0}(x, y) * S_{\mathrm{H}}(x, y, z) \\
& =\mathfrak{F}^{-1}\left[\mathfrak{F}\left(E_{0}\right) \cdot \mathfrak{F}\left(S_{\mathrm{H}}\right)\right]
\end{aligned}
$$

where $S_{\mathrm{H}}$ is the Huygens PSF

$$
S_{\mathrm{H}}(x, y, z)=-\frac{\mathrm{i} k}{2 \pi z} \exp \left[\mathrm{i} k \sqrt{x^{2}+y^{2}+z^{2}}\right] .
$$

The whole process requires three Fourier transforms, which are carried out using the FFT algorithm. The pixel sizes

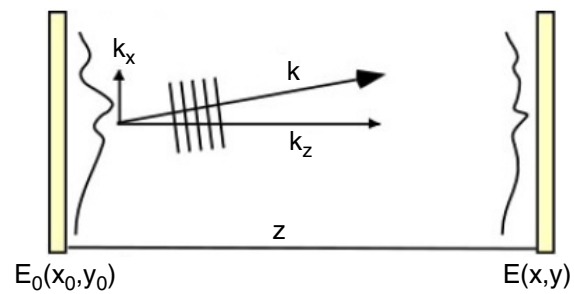

Figure 2. Coordinate system for reconstruction of the hologram in the angular spectrum method.

of the images reconstructed by the convolution approach are equal to that of the hologram. In order to achieve as high a lateral resolution as possible, one keeps the object-hologram distance as short as possible, but the discrete Fourier transform necessitates a minimum distance such that

$$
z_{\min }=\frac{a_{x}^{2}}{n_{x} \lambda}
$$

where $a_{x}=n_{x} \Delta x$ is the size of the hologram and $n_{x}$ and $\Delta x$ are the number and size of pixels. At too close a distance, the spatial frequency of the hologram is too low and aliasing occurs. Normally the object is placed just outside this minimum distance.

\subsection{Fresnel transform method}

The Fresnel transformation [3] is the most commonly used method in holographic reconstruction, because of the computational efficiency. The approximation of a spherical Huygens wavelet by a parabolic surface allows the calculation of the diffraction integral using a single Fourier transform. The PSF can be simplified by the Fresnel approximation as

$$
S(x, y ; z)=-\frac{\mathrm{i} k}{2 \pi z} \exp \left[\mathrm{i} k z+\mathrm{i} \frac{k}{2 z}\left(x^{2}+y^{2}\right)\right]
$$

and the reconstructed wavefield is

$$
\begin{gathered}
E(x, y ; z)=-\frac{\mathrm{i} k}{2 \pi z} \exp \left[\mathrm{i} k z+\frac{\mathrm{i} k}{2 z}\left(x^{2}+y^{2}\right)\right] \\
\times \iint E_{0}\left(x_{0}, y_{0}\right) \exp \left[\frac{\mathrm{i} k}{2 z}\left(x_{0}^{2}+y_{0}^{2}\right)\right] \\
\quad \times \exp \left[-\frac{\mathrm{i} k}{z}\left(x x_{0}+y y_{0}\right)\right] \mathrm{d} x_{0} \mathrm{~d} y_{0} \\
=\exp \left[\frac{\mathrm{i} k}{2 z}\left(x^{2}+y^{2}\right)\right] \mathfrak{F}\left[E_{0} \cdot S\right] .
\end{gathered}
$$

The resolution $\Delta x$ of the reconstructed images determined directly from the Fresnel diffraction formula will vary as a function of the reconstruction distance $z$ as

$$
\Delta \alpha=\frac{\lambda z}{N \Delta x_{0}}
$$

where $N$ is the number of pixels and $\Delta x_{0}$ is the pixel width of the CCD camera. As with the Huygens convolution method there is a minimum $z$ distance requirement set by equation (4). 

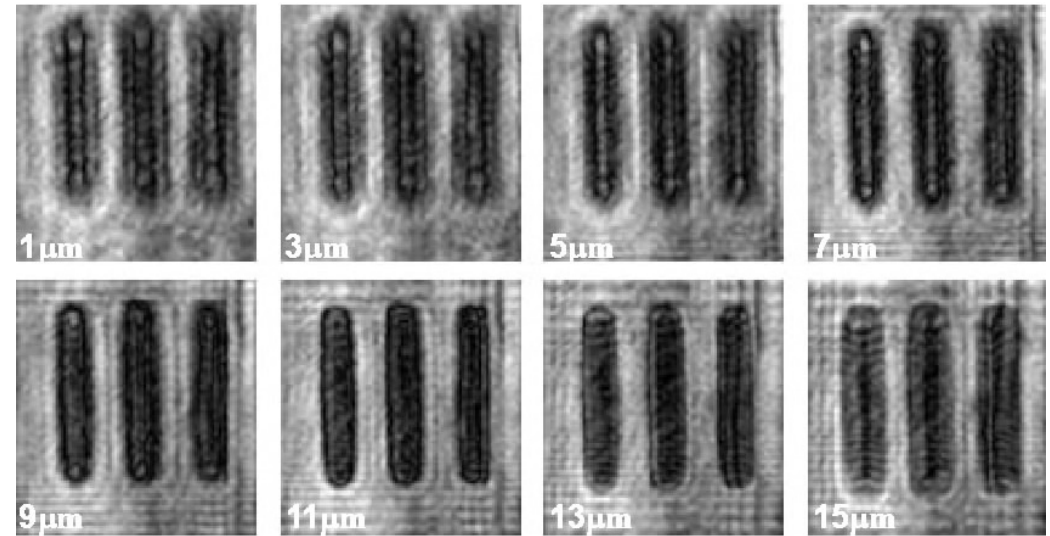

Figure 3. Numerical focusing in digital holography of an element of a USAF 1951 resolution target from a single hologram. Images are of a $30 \times 30 \mu \mathrm{m}^{2}$ area $(360 \times 360$ pixels) with $z$ scanned from 1 to $15 \mu \mathrm{m}$ in steps of $2 \mu \mathrm{m}$.
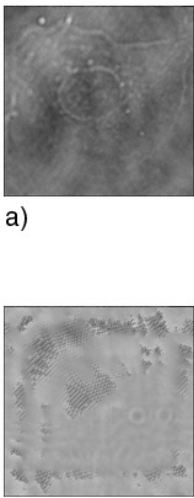

f)

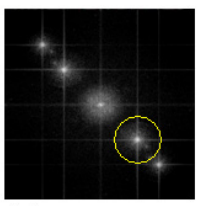

b)

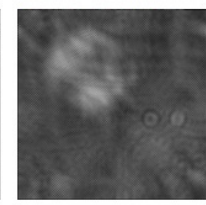

g)
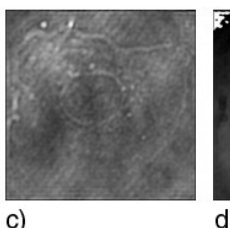

d)
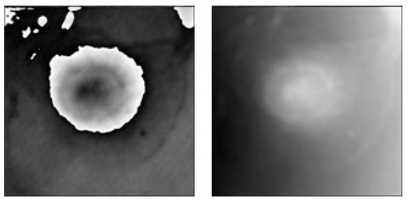

e)

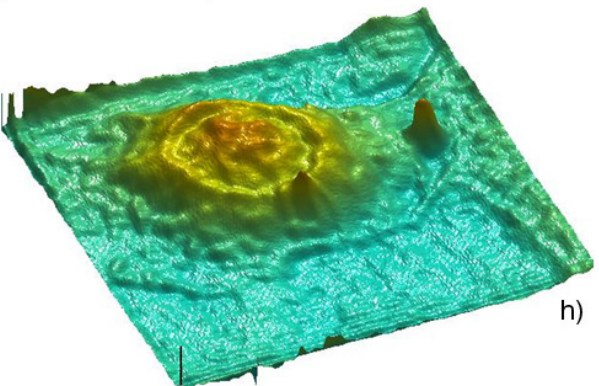

Figure 4. Holography of a SKOV ovarian cancer cell. The image area is $60 \times 60(424 \times 424$ pixels $)$ and the image is at $z=1.0 \mu \mathrm{m}$ from the hologram: (a) hologram; (b) angular spectrum; (c) amplitude and (d) phase images by the angular spectrum method; (e) unwrapped phase image of (d); (f) amplitude and (g) phase images by the Huygens convolution method. (h) 3D perspective rendering of (e).

\subsection{Angular spectrum method}

The angular spectrum method [16] is seen to be fairly efficient computationally but with the highest degree of accuracy. Refering to figure 2 , if $E_{0}\left(x_{0}, y_{0} ; 0\right)$ is the wavefield at plane $z=0$, the angular spectrum $A\left(k_{x}, k_{y} ; 0\right)$ at this plane is obtained by taking the Fourier transform:

$$
\begin{aligned}
& A\left(k_{x}, k_{y} ; 0\right)=\iint E_{0}\left(x_{0}, y_{0} ; 0\right) \\
& \quad \times \exp \left[-\mathrm{i}\left(k_{x} x_{0}+k_{y} y_{0}\right)\right] \mathrm{d} x_{0} \mathrm{~d} y_{0}
\end{aligned}
$$

where $k_{x}$ and $k_{y}$ are corresponding spatial frequencies of $x$ and $y$. Fourier-domain filtering can be applied to the spectrum to block unwanted spectral terms in the hologram and select a region of interest corresponding only to the object spectrum. A modified wavefield $\bar{E}_{0}\left(x_{0}, y_{0} ; 0\right)$ can be written as the inverse Fourier transform of the filtered angular spectrum $\bar{A}\left(k_{x}, k_{y} ; 0\right)$,

$\bar{E}_{0}\left(x_{0}, y_{0} ; 0\right)=\iint \bar{A}\left(k_{x}, k_{y} ; 0\right) \exp \left[\mathrm{i}\left(k_{x} x_{0}+k_{y} y_{0}\right)\right] \mathrm{d} k_{x} \mathrm{~d} k_{y}$.
The angular spectrum at plane $z, A\left(k_{x}, k_{y} ; z\right)$ is calculated from $\bar{A}\left(k_{x}, k_{y} ; 0\right)$, with $k_{z}=\sqrt{k^{2}-k_{x}^{2}-k_{y}^{2}}$

$$
A\left(k_{x}, k_{y} ; z\right)=\bar{A}\left(k_{x}, k_{y} ; 0\right) \exp \left[\mathrm{i} k_{z} z\right] .
$$

The reconstructed complex wavefield of any plane perpendicular to the propagating $z$ axis is found by

$$
\begin{gathered}
E(x, y ; z)=\iint A\left(k_{x}, k_{y} ; z\right) \exp \left[i\left(k_{x} x+k_{y} y\right)\right] \mathrm{d} k_{x} \mathrm{~d} k_{y} \\
=\mathfrak{F}^{-1}\left\{\operatorname{filter}\left[\mathfrak{F}\left\{E_{0}\right\}\right] \exp \left[\mathrm{i} k_{z} z\right]\right\} .
\end{gathered}
$$

Here 'filter' represents filtering in the spectral domain. Two Fourier transforms are needed for the calculation in comparison to the one needed by the Fresnel transform. However, once the field is known at any one plane, only one additional Fourier transform is needed to calculate the field at different values of $z$. This method allows frequency-domain spectrum filtering to be applied, which for example can be used to block or remove the disturbance of the zero order and twin image component. A significant advantage of the angular spectrum is that there is no minimum $z$ distance requirement. 


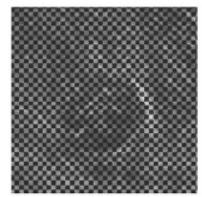

a)

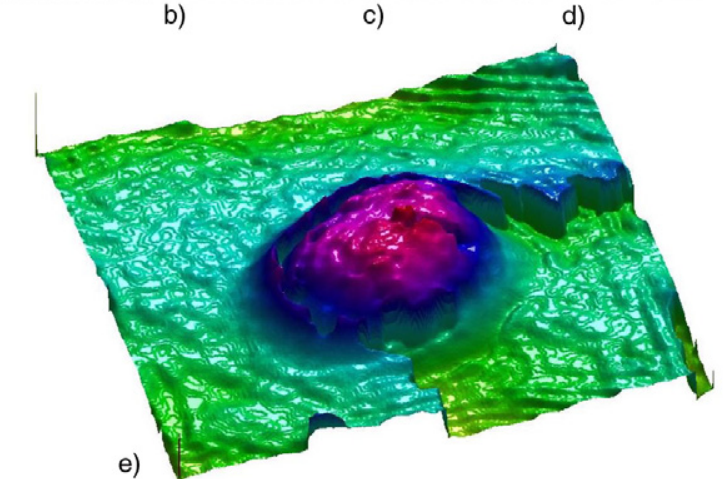

Figure 5. Holography of an onion nucleus. The image area is $30 \times 30 \mu \mathrm{m}^{2}(436 \times 436$ pixels $)$ and the image is at $z=22 \mu \mathrm{m}$ from the hologram: (a) hologram; (b) holographic amplitude and (c) phase images; (d) unwrapped phase image; (e) 3D pseudo-colour rendering of (d).

\subsection{Numerical focusing}

The optical field can be calculated at any number of image planes from a single hologram, emulating the mechanical focusing control of a conventional microscope. To illustrate this focusing ability we show a sequence of eight images in figure 3 calculated in the range of $z=1-15 \mu \mathrm{m}$ in steps of $2 \mu \mathrm{m}$. Each image is a $30 \times 30 \mu \mathrm{m}^{2}$ area of a resolution target. As the focus is scanned, one observes the bars move into focus as it passes through the various image planes.

\section{Quantitative phase imaging}

Figure 4 illustrates the implementation of the numerical algorithms in the reconstruction of a SKOV3 ovarian cancer cell. The area is $60 \times 60 \mu \mathrm{m}^{2}$ with $424 \times 424$ pixels. Figure 4 (a) is the holographic interference pattern recorded by the CCD camera, and its Fourier transform in figure 4(b) is the angular spectrum. It contains both the zero order and twin images, as well as artifact due to stray interference components. The virtual image component, the highlighted circular area, is selected. A propagation phase factor $(z=1.0 \mu \mathrm{m})$ is multiplied, and finally inverse-Fourier transformed to obtain the amplitude image in figure 4(c) and the phase image in figure 4(d). The physical thickness of the cell can be calculated from

$$
d=\lambda(\Delta \varphi / 2 \pi) /\left(n-n_{0}\right)
$$

where $\lambda$ is the wavelength, $\Delta \varphi$ is the phase step and $\left(n-n_{0}\right)$ is the index difference between the film and air. For example, the layer of lamellipodia around the edge of the cell is found to be about $110 \mathrm{~nm}$, assuming $n=1.375$ for the cell. The phase map is rendered in pseudo-coloured 3D perspective in figure 4(h). Especially notable in the phase map is the lack of the coherent noise conspicuous in the amplitude image and prevalent in most other holographic imaging methods. The amplitude and phase images obtained from the Huygens convolution method

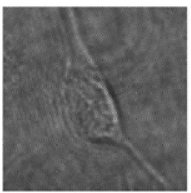

a)

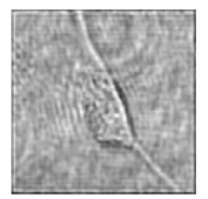

b)

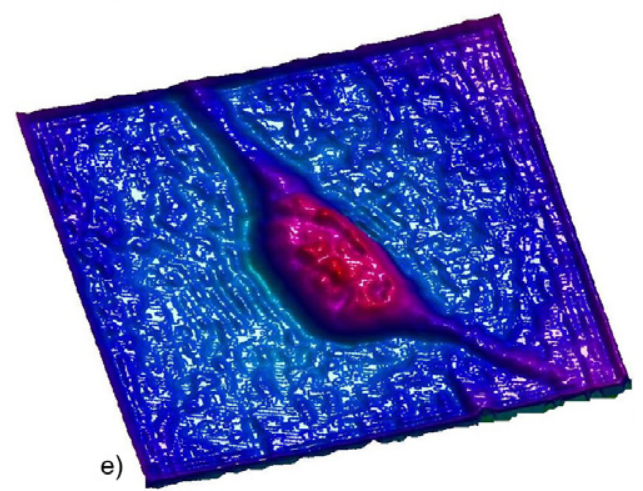

Figure 6. Holography of a living SKOV ovarian cancer cell. The image area is $70 \times 70 \mu \mathrm{m}^{2}(448 \times 448$ pixels $)$ and the image is at $z=12 \mu \mathrm{m}$ from the hologram: (a) hologram; (b) amplitude and (c) phase images; (d) unwrapped phase image; (e) 3D perspective rendering of $(d)$

are shown in figures 4(f) and (g), while those obtained from the Fresnel method are omitted because they are completely scrambled. The main reason for the obvious degradation of these images is the insufficient off-axis angle at such short $z$ distance to separate out the zero-order component.

Figure 5 shows digital holography of an onion nucleus. The panels display the (a) hologram, (b) amplitude image, (c) phase image, and (d) phase image unwrapped by a software algorithm. Pseudo-colour 3D rendering of (d) is shown in (e). The image size is $30 \times 30 \mu \mathrm{m}^{2}$ with $436 \times 436$ pixels. The phase image is a clear view of the optical thickness variation of the nucleus in the middle of the body of the cell. In figure 6 we show digital holography of a living SKOV ovarian cancer cell. The image demonstrates the high quality that can be obtained, displaying the nuclear membrane. The lamellipodia of the cell are seen to extend out in order to occupy a large area as it attempts to migrate.

\section{Dual-wavelength imaging}

A difficulty in both interferometry and phase imaging is the $2 \pi$ ambiguity. A number of phase unwrapping algorithms have been developed to remove these and improve the quality and interpretation of the image. However, these often require both substantial user intervention and the level of phase noise and phase discontinuity to lie within strict limits. It has been demonstrated that the wavelength can be extended to that of a synthetic or beat wavelength $\Lambda_{12}=\lambda_{1} \lambda_{2} /\left|\lambda_{1}-\lambda_{2}\right|$, by the use of two wavelengths at $\lambda_{1}$ and $\lambda_{2}$ [18]. By generation and combination of two phase maps using two or more separate wavelengths, the phase ambiguities and discontinuities which exist in the image can be removed. The procedure involves subtraction of two phase maps $\phi_{1}$ and $\phi_{2}$ derived from $\lambda_{1}$ and $\lambda_{2}$, such that $\phi_{12}=\phi_{1}-\phi_{2}$. Adding $2 \pi$ wherever $\phi_{12}<0$ yields a new 'coarse' phase map with a longer range free of 

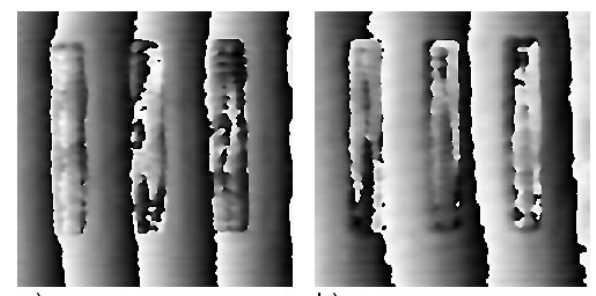

a)

b)

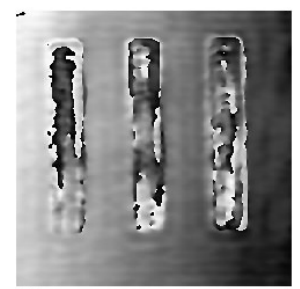

c)

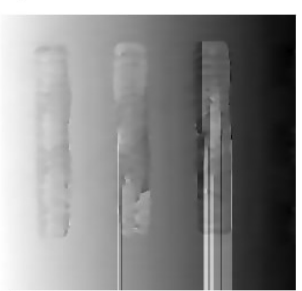

d)

Figure 7. (a) The wrapped phase map reconstructed from the hologram at the green wavelength $\lambda_{1}=0.532 \mu \mathrm{m}$ and (b) the red wavelength at $\lambda_{2}=0.633 \mu \mathrm{m}$; (c) the fine map obtained by the phase maps shown in (a) and (b); (d) is the unwrapped phase map by a software program.

discontinuities and extended axial range. By proper choice of two wavelengths it can be seen that the axial range $\Lambda_{12}$ can be adjusted to any value that would fit the axial size of the object being imaged. However, a drawback is that any phase noise in each single-wavelength phase map is amplified by a factor equal to the magnification of the wavelengths. The final step in the procedure is to reduce the noise back to that of the level of the single-wavelength phase maps. This is performed by dividing the coarse map by integer multiples of $\lambda_{1}$ and pasting on the single-wavelength phase map to obtain the fine map.

An example of dual-wavelength phase imaging digital holography is illustrated in figure 7 . The combination of the phase maps by the green wavelength $\lambda_{1}=0.532 \mu \mathrm{m}$ figure 7(a), and the red wavelength $\lambda_{2}=0.633 \mu \mathrm{m}$, figure 7(b), produces a fine map with a new larger beat wavelength $\Lambda_{12}=3.33 \mu \mathrm{m}$, figure 7(c). Some discontinuities still remain in the image as the bars contain a large fluctuation of phase due to the small amount of signal that is obtained from these areas. The software implemented phase unwrapping algorithm in figure 7(d) has a defect that propagates beyond the noisy regions.

\section{Wavelength-scanning digital interference holography (WSDIH)}

In wavelength-scanning digital interference holography, a number of holograms are recorded using a range of sequential intervals of wavelength [19]. The holograms are subsequently reconstructed and the numerical superposition of reconstructed holographic images results in a tomographic image with a synthesized short coherence length and corresponding axial resolution of around $10 \mathrm{~nm}$. In contrast to the application of other commonly used three-dimensional imaging techniques such as confocal microscopy and optical coherence tomography, WSDIH does not involve mechanical scanning of the three-dimensional volume and still achieves a comparable resolution.
For $N$ different wavelengths, the reconstructed fields are all superposed. The resultant wavefield at $r$ is

$$
\begin{aligned}
E(\mathbf{r}) & \sim \sum_{k} \int A\left(\mathbf{r}_{\mathrm{P}}\right) \exp \left(\mathrm{i} k\left|\mathbf{r}-\mathbf{r}_{\mathbf{P}}\right|\right) \mathrm{d}^{3} \mathbf{r}_{\mathbf{P}} \\
& \sim \int A\left(\mathbf{r}_{\mathrm{P}}\right) \delta\left(\mathbf{r}-\mathbf{r}_{\mathbf{P}}\right) \mathrm{d}^{3} \mathbf{r}_{\mathbf{P}} \\
& \sim A(\mathbf{r})
\end{aligned}
$$

where $r_{\mathrm{p}}$ represents the position of a point $\mathrm{P}$ which scatters the illuminating beam and $A(r)$ is the wavefield at the object position. For $N$ number of wavelengths at regular intervals $\Delta(1 / \lambda)$, the object field $A(\mathbf{r})$ repeats itself with beat wavelength $\Lambda=[\Delta(1 / \lambda)]^{-1}$ and axial resolution $\delta=\Lambda / N$. By choosing appropriate values of $\Delta(1 / \lambda)$ and $N$, the beat wavelength $\Lambda$ can be matched to the axial extent of the object, and $\delta$ to the desired level of axial resolution.

Figure 8 illustrates the increase in axial resolution by numerical superposition of the reconstructed holographic images using a sequential number of wavelengths in the range of 575.0-605.0 nm in 20 steps. The five frames in the figure are with one, two, four, eight, and 20 wavelengths superposed. As the synthesized coherence length decreases to $\delta=12 \mu \mathrm{m}$ the contour widths become narrower. Figure 9 is the result of a DIH imaging experiment on a $2.62 \times 2.62 \mathrm{~mm}^{2}$ area of a piece of beef tissue. Here we have used wavelengths in the range of $585.0-599.0 \mathrm{~nm}$ at 31 steps so that the axial range is $\Lambda=750 \mu \mathrm{m}$ and the axial resolution $\delta=25 \mu \mathrm{m}$. The specimen is a thin layer of beef tissue pressed to about $1.5 \mathrm{~mm}$ thickness on a slide glass and otherwise exposed to air. The images in figure 9(a) show tissue layers at several depths up to about $500 \mu \mathrm{m}$ below the surface. Much of the reflection signal is apparently from the tissue-air and tissueglass interface. One can discern the striation of muscle fibre bundles. Figure 9(b) shows variations of the tissue layers in a few $x-z$ cross-sectional images.

\section{Conclusion}

Interference techniques in digital holography are discussed and experimental results from each technique are presented. Numerical reconstruction algorithms commonly used in digital holography are reviewed and their application to a test object is investigated. The angular spectrum algorithm is seen to be a particularly advantageous method of holographic reconstruction. Frequency-domain spectrum filtering can be applied, which can for example be used to remove noise and background terms. Also, there is no requirement for a minimum $z$ distance, as in the commonly used Huygens convolution and Fresnel transform reconstruction methods. Furthermore, we demonstrate the application of the angular spectrum in obtaining high quality images of biological objects with quantitative phase analysis. On the other hand, a common problem in phase imaging digital holography is the $2 \pi$ ambiguity. The dual-wavelength technique offers a convenient and attractive alternative to using a software based phase unwrapping algorithm. The advantage of the multi-wavelength technique is clearly demonstrated when unwrapping an object that does not fulfil the strict requirements of the unwrapping algorithm. Finally, we show the application of wavelength-scanning digital interference holography in 

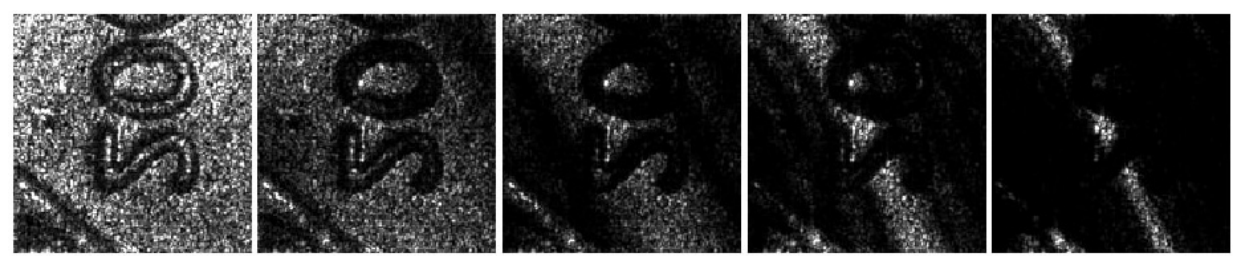

Figure 8. Build-up of axial resolution by superposition of holographic images of a penny using a range of wavelengths with $N=1,2,4,8$, and 20.

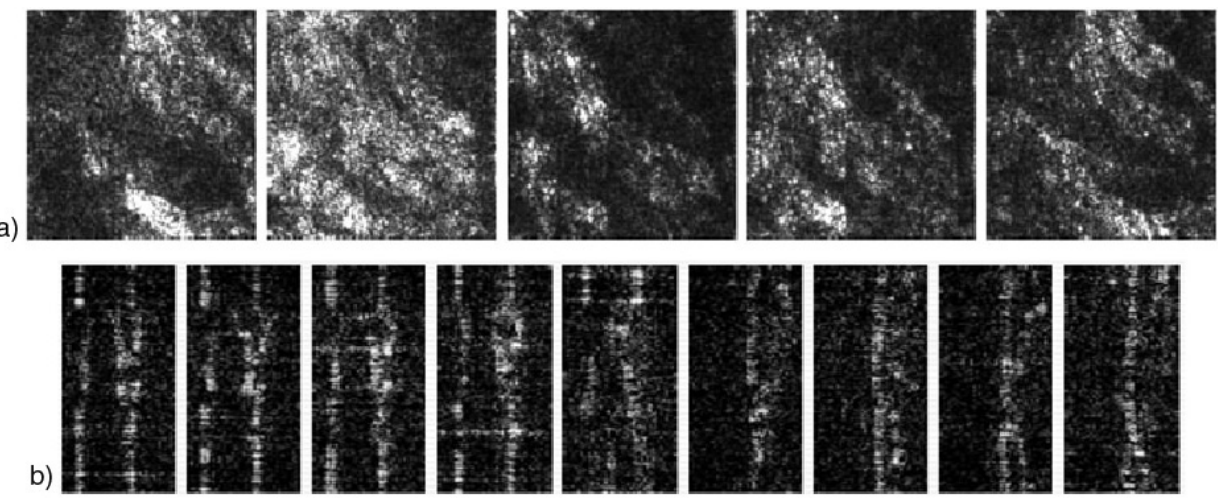

Figure 9. WSDIH tomography of beef tissue. The image volume is $2.62 \mathrm{~mm} \times 2.62 \mathrm{~mm} \times 750 \mu \mathrm{m}, \lambda=585.0-599.0 \mathrm{~nm}$ and $N=31$, so that $\Lambda=750 \mu \mathrm{m}$ and $\delta=25 \mu \mathrm{m}$. (a) $x-y$ transverse images at several depths. (b) $x-z$ cross-sectional images displaying variations of tissue layers across the field.

obtaining tomographic images with a synthesized short coherence length and axial resolution of around $10 \mu \mathrm{m}$. The techniques presented in this paper demonstrate the effectiveness of digital holography for biomedical microscopy applications. In particular, the phase imaging digital holography has great potential to provide quantitative phase images of transparent biological specimens with nanometre resolution of optical thickness variations. The wavelengthscanning digital interference holography is being developed for tomographic imaging of epithelial tissues.

\section{Acknowledgment}

This work is supported in part by the National Science Foundation.

\section{References}

[1] Gabor D 1948 A new microscopic principle Nature 161 777-8

[2] Leith E N and Upatnieks J 1962 Reconstructed wavefronts and communication theory J. Opt. Soc. Am. $521123-30$

[3] Schnars U and Jueptner W P 1994 Direct recording of holograms by a CCD target and numerical reconstruction Appl. Opt. 33 179-81

[4] Schnars U and Jueptner W P 2002 Digital recording and numerical reconstruction of holograms Meas. Sci. Technol. 13 R85-101

[5] Grilli S, Ferraro P, De Nicola S, Finizio A, Pierattini G and Meucci R 2001 Whole optical wave fields reconstruction by digital holography Opt. Express 9 294-302

[6] Cuche E, Bevilacqua F and Depeursinge C 1999 Digital holography for quantitative phase-contrast imaging $O p t$. Lett. 24 291-3

[7] Cuche E, Marquet P and Depeursinge C 1999 Simultaneous amplitude-contrast and quantitative phase-contrast microscopy by numerical reconstruction of Fresnel off-axis holograms Appl. Opt. 38 6994-7001
[8] Xu M L, Peng X, Miao J and Asundi A 2001 Studies of digital microscopic holography with applications to microstructure testing Appl. Opt. 40 5046-51

[9] Pedrini G and Tiziani H J 1997 Quantitative evaluation of two-dimensional dynamic deformations using digital holography Opt. Laser Technol. 29 249-56

[10] Picart P, Leval J, Mounier D and Gougeon S 2005 Some opportunities for vibration analysis with time averaging in digital Fresnel holography Appl. Opt. 44 337-43

[11] Xu W, Jericho M H, Meinertzhagen I A and Kreuzer H J 2001 Digital in-line holography for biological applications Proc. Natl Acad. Sci. USA 98 11301-5

[12] Popescu G, Delflores L P, Vaughan J C, Badizadegan K, Iwai H, Dasari R and Feld M S 2004 Fourier phase microscopy for investigation of biological structures and dynamics Opt. Lett. 29 2503-5

[13] Marquet P, Rappaz B, Magistretti P J, Cuche E, Emery Y, Colomb T and Depeursinge C 2005 Digital holographic microscopy: a noninvasive contrast imaging technique allowing quantitative visualization of living cells with subwevelength axial accuracy Opt. Lett. 30 468-70

[14] Carl D, Kemper B, Wernicke G and Von Bally G 2004 Parameter-optimized digital holographic microscope for high-resolution living-cell analysis Appl. Opt. 43 6536-44

[15] Mann C J, Yu L, Lo C M and Kim M K 2005 High-resolution quantitative phase-contrast microscopy by digital holography Opt. Express 13 8693-8

[16] Yu L and Kim M K 2005 Wavelength-scanning digital interference holography for tomographic 3D imaging using the angular spectrum method Opt. Lett. 302092

[17] Demetrakopoulos T H and Mittra R 1974 Digital and optical reconstruction of images from suboptical diffraction patterns Appl. Opt. 13 665-70

[18] Gass J, Dakoff A and Kim M K 2003 Phase imaging without $2 \pi$ ambiguity by multiple wavelength digital holography Opt. Lett. 28 1141-3

[19] Kim M K 2000 Tomographic three-dimensional imaging of a biological specimen using wavelength-scanning digital interference holography Opt. Express 7 305-10 